United Nations Educational Scientific and Cultural Organization

and

International Atomic Energy Agency

THE ABDUS SALAM INTERNATIONAL CENTRE FOR THEORETICAL PHYSICS

\title{
RATIONAL SURFACES HAVING ONLY A FINITE NUMBER OF EXCEPTIONAL CURVES
}

\author{
Mustapha Lahyane ${ }^{1}$ \\ The Abdus Salam International Centre for Theoretical Physics, \\ Trieste, Italy.
}

\begin{abstract}
We characterize the rational surfaces $X$ which have a finite number of $(-1)$-curves under the assumption that $-K_{X}$ is nef (i.e., the intersection number of $K_{X}$ with any effective divisor on $X$ is less than or equal to zero, where $K_{X}$ is a canonical divisor on $X$ ) and having self-intersection zero. A ( -1$)$-curve is a smooth rational curve of self-intersection -1 .
\end{abstract}

MIRAMARE - TRIESTE

October 2001

\footnotetext{
${ }^{1}$ E-mail: lahyanem@ictp.trieste.it
} 


\section{Introduction}

By a surface we mean here a compact complex analytic manifold of complex dimension two. According to the curves on surfaces, one can distinguish between three classes: there are those which have only a finite number of curves, i.e. the surfaces having algebraic dimension zero; those which have curves but not enough, i.e. the surfaces having algebraic dimension one; and those which are rich in curves, i.e. the projective ones. For more details see [5, Theorems 3.1, $4.1,4.2,4.3,5.1]$, [6], and [12].

We restrict ourselves to curves which are smooth, rational and of self-intersection -1 (such curves are called (-1)-curves), and ask which surfaces have an infinite number of (-1)-curves. The answer is that only rational surfaces may have an infinite number of $(-1)$-curves (e.g., see [11]).

In this paper we give a characterization of rational surfaces which have a finite number of $(-1)$-curves under the assumption that an anti-canonical divisor of the surface is nef (see the definition in the next paragraph) and of self-intersection zero.

Let $X$ be a smooth projective rational surface. From now on we assume that $-K_{X}$ is nef (i.e., the intersection number of the divisor $K_{X}$ with any effective divisor on $X$ is less or equal to zero, where $K_{X}$ is a canonical divisor on $X$ ) and of self-intersection zero.

It is easy to see that $X$ is a blowing-up nine points (possibily infinitely near) of the complex projective plane.

According to the position of the nine points, $X$ may have a finite or an infinite number of (-1)-curves.

Masayoshi Nagata ([10], proposition 6a., p.282) proved that if the nine points are in general position, then $X$ has an infinite number of $(-1)$-curves (i.e., smooth rational curves of selfintersection -1 ).

Ulf Persson and Rick Miranda ([9]) studied the case when the nine points are the base points of a linear system of plane cubics without fixed components. In this case $X$ is an elliptic surface with a section. They classified all such surfaces which have a finite number of $(-1)$-curves and called them extremal jacobian elliptic rational surfaces. For each case, they gave the number of $(-1)$-curves.

We will use the following notations:

$\sim$ the linear equivalence of divisors on $X$;

$[D]$ the set of divisors $D^{\prime}$ on $X$ such that $D^{\prime} \sim D$;

$\operatorname{Div}(X)$ the group of divisors on $X$;

$N S(X)$ the group quotient $\frac{\operatorname{Div}(X)}{\sim}$ of $\operatorname{Div}(X)$ by $\sim$ (the linear, algebraic and numerical equivalences are the same on $\operatorname{Div}(X)$ since $X$ is a rational surface);

$D . D^{\prime}$ will denote the intersection number of the divisor $D$ with the divisor $D^{\prime}$, in particular the self-intersection of $D$ is $D^{2}=D . D$; 
$\bar{D}$ the associated element to the divisor $D$ in the tensor product of the group $N S(X)$ with the field of rational numbers over the ring of integers.

Following [9], we will define a smooth projective rational surface having a finite number of $(-1)$-curves on it an extremal rational surface. Our main result gives a classification of extremal rational surfaces:

Theorem 1.1 Let $X$ be a smooth projective rational surface having $-K_{X}$ nef and of selfintersection zero. Then the following are equivalent:

1. $X$ is extremal.

2. X satisfy the two conditions below:

a. the rank of the matrix $\left(C_{i} . C_{j}\right)_{i, j=1, \ldots, r}$ is equal to 8 , where $\left\{C_{i} ; i=1, \ldots, r\right\}$ is the finite set of $(-2)$-curves on $X$; a $(-2)$-curve is a smooth rational curve of selfintersection -2 .

b. There exist $r$ strictly positive rational numbers $a_{i}, i=1, \ldots, r$ such that $-\bar{K}_{X}=$ $\sum_{i=1}^{i=r} a_{i} \bar{C}_{i}$.

This paper is organized as follows. In most cases $X$ denotes a smooth projective rational surface defined over the field of complex numbers $\mathbb{C}$ such that its anti-canonical class is nef and of self-intersection zero. In Sect. 2 we introduce some well-known facts about smooth rational surfaces. In Sect. 3 we prove that the existence of a family of $(-2)$-curves on $X$ such that its elements are linearly dependents in the tensor product $N S(X)_{\mathbb{Q}}$ of the Néron-Severi group $N S(X)$ of $X$ and the field of rational numbers $\mathbb{Q}$ over the ring of integers $\mathbb{Z}$ gives an elliptic structure to $X$ (see proposition $3.5, \mathrm{p} .10$ ); As a corollary, the number of $(-2)$-curves on $X$ is finite and bounded by the optimal integer 12 (see corollary 3.6, p.10). In Sect. 4 we give a useful criterion (see proposition 4.2, p.12) for the existence of an infinite number of $(-1)$-curves on the surface. In Sect. 5 we give the proof of theorem 1.1.

\section{Preliminaries}

In this section we fix our notations and gather some well-known general facts concerning smooth projective rational surfaces.

\subsection{Notation and First Properties}

Let $X$ be a smooth projective rational surface defined over the field of complex numbers $\mathbb{C}$. Recall that the Néron-Severi group $N S(X)$ of $X$ is the group of divisor classes on $X$ modulo algebraic equivalence. The following facts are well-known: 
- $N S(X)$ is finitely generated abelien group of rank $\rho(X)(\rho(X)$ is called the Picard number of $X)$;

- $N S(X)$ is torsion free abelien group;

- $\rho(X)=10-K_{X}^{2}$, where $K_{X}$ represent a canonical divisor on $X$;

- $N S(X)$ is equipped with a symmetric bilinear form induced from the intersection form defined on the set of divisors $\operatorname{Div}(X)$ on $X$; this symmetric bilinear form will be called from now on the intersection form on $N S(X)$;

- $N S_{\mathbb{Q}}(X)$ is by definition the tensor product of $N S(X)$ and the field of rational numbers $\mathbb{Q}$ over the ring of integers $\mathbb{Z}$,i.e., $N S_{\mathbb{Q}}(X)=N S(X) \otimes_{\mathbb{Z}} \mathbb{Q}$;

- $N S_{\mathbb{Q}}(X)$ is equipped with a symmetric bilinear form induced by the intersection form on $N S(X)$; this symmetric bilinear form will be called from now on the intersection form on $N S_{\mathbb{Q}}(X)$.

If $D$ is a divisor on $X$, we adopt the following notations:

- $[D]$ its equivalence class in $N S(X)$;

- $\bar{D}$ is the unique element associated to $[D]$ in $N S_{\mathbb{Q}}(X)$.

By definition:

- $K_{X}$ (resp. $\left.-K_{X}\right)$ denotes a canonical (resp. anti-canonical) divisor on the surface $X$;

- $\left[K_{X}\right]$ (resp. $\left[-K_{X}\right]$ ) is the canonical (resp. anti-canonical) class of $X$;

- $K_{X}^{\perp}$ is the orthogonal of $\bar{K}_{X}$ in $N S_{\mathbb{Q}}(X)$, and by abuse of notation, the orthogonal of $\left[K_{X}\right]$ in $N S(X)$ is also denoted by $K_{X}^{\perp}$.

The intersection form defined on $\operatorname{Div}(X)$ (resp. on $N S(X)$, resp. on $N S_{\mathbb{Q}}(X)$ ) is noted by “.". In particular for any two divisors $D$ and $D^{\prime}$ on $X$, the equalities hold: $D \cdot D^{\prime}=\bar{D} \cdot \overline{D^{\prime}}=$ $[D] .\left[D^{\prime}\right]$; if $D=D^{\prime}$, the intersection number of $D$ and $D^{\prime}$ is called the self-intersection of the divisor $D$ and is denoted by $D^{2}$.

Similarly for every element $x$ of $N S_{\mathbb{Q}}(X)$, the rational number $x . x$ is denoted by $x^{2}$ and we say that it is the self-intersection of $x$.

The following well-known lemma is useful:

Lemma 2.1 Let $X$ be a smooth projective rational surface such that the self-intersection of its canonical divisor $K_{X}$ is zero. Then the intersection form is negative semi-definite on $K_{X}^{\perp}$; and for every element $x \in K_{X}^{\perp}$, the following equivalence hold: 


$$
x^{2}=0 \text { if and only if } x=r \bar{K}_{X} \text { for some rational number } r .
$$

We give here an elementary proof. Let $x$ be an element of $K_{X}^{\frac{1}{X}}$, we can suppose that $x$ is the divisor class of a divisor $D$ on $X$. If $D^{2}$ is strictly larger than zero, then by using the index theorem ([4]), $K_{X}$ would be either of self-intersection strictly less that zero or numeriquely trivial. these are impossible since $X$ is rational and $K_{X}^{2}=0$.

Let $A$ be an ample divisor on $X$, and consider the divisor $E$ defined as $E=A . K_{X} D-A . D K_{X}$ . We have $E^{2}=0=A$. $E$.

Hence, by the index theorem ([4]), the divisor $A \cdot K_{X} D$ is numeriquely equivalent to A.D $K_{X}$. Since $X$ is rational and $-K_{X}$ is an effective divisor, we obtain that $\bar{D}=\frac{A \cdot D}{A \cdot K_{X}} \overline{K_{X}}$.

\subsection{Riemann-Roch theorem for Rational Surfaces}

Let $X$ be a smooth projective surface, its canonical divisor is denoted by $K_{X}$. If $D$ is a divisor on $X$, then:

- $O_{X}(D)$ is the invertible sheaf associated to $D$;

- for $i=0,1,2 \quad: \quad H^{i}\left(X, O_{X}(D)\right)$ is the $i^{\text {th }}$ cohomology group of the invertible sheaf $O_{X}(D)$;

- for $i=0,1,2: \quad h^{i}\left(X, O_{X}(D)\right)$ is the dimension of $H^{i}\left(X, O_{X}(D)\right)$.

Definition 2.2 $A$ divisor $D$ on a smooth projective surface $X$ is effective if $n_{i} \geq 0$ for all $i=1, \ldots, r$, where $r$ is a non-negative integer and where $D=n_{1} C_{1}+\ldots+n_{r} C_{r}$ (the $C_{i}$, $i=1, \ldots, r$ are reduced irreducible curves on $X)$.

An element of $N S(X)$ is effective if it is the class of an effective divisor on $X$.

A proof of the next lemma is given in [4, proposition 7.7, p. 157]

Lemma 2.3 An elemnet $[D]$ of $N S(X)$ is effective if and only if the integer $h^{0}\left(X, O_{X}(D)\right)$ is not equal to zero.

It is easy to see that:

Lemma 2.4 If $D$ is an effective divisor on a smooth projective rational surface $X$, then the integer $h^{2}\left(X, O_{X}(D)\right)$ is equal to zero.

Definition 2.5 Let $D$ be a divisor on a smooth projective surface X. The Euler-Poincaré characteristic $\chi(D)$ of $D$ is the following integer:

$$
h^{0}\left(X, O_{X}(D)\right)-h^{1}\left(X, O_{X}(D)\right)+h^{2}\left(X, O_{X}(D)\right),
$$

where $O_{X}(D)$ is the invertible sheaf associated to $D$. This integer is also called the EulerPoincaré characteristic of the sheaf $O_{X}(D)$ (resp. of the class $[D]$ of $D$ in the Néron-Severi group $N S(X)$ of $X)$ associated to the divisor $D$. 
The Riemann-Roch theorem is:

Theorem 2.6 Let $D$ be a divisor on a smooth projective rational surface $X$, the Euler-Poincaré characteristic $\chi(D)$ of the divisor $D$ is given by:

$$
\chi(D)=1+\frac{1}{2}\left(D^{2}-K_{X} \cdot D\right)
$$

Definition 2.7 A smooth projective rational surface is anti-canonical if the anti-canonical class of this surface is effective.

It is easy to see:

Lemma 2.8 Every smooth rational surface for which the self-intersection of its canonical class is larger or equal to zero is anti-canonical.

The adjunction formula is:

Lemma 2.9 Let $Y$ be a smooth projective surface. For every irreducible curve $C$ on $Y$, the equality hold:

$$
C^{2}+C \cdot K_{Y}=2 p_{a}(C)-2
$$

where $K_{Y}$ is a canonical divisor on $Y$ and $p_{a}(C)$ is the arithmetic genus of the curve $C$.

Definition 2.10 Let $X$ be a smooth projective surface.

- $A$ divisor $D$ on $X$ is nef if and only if D.C $\geq 0$ for every irreducible curve $C$ on $X$.

- An element of $N S(X)$ is nef if it is the class of a nef divisor on $X$.

Using the Riemann-Roch theorem and the adjunction formula, one can obtain:

Lemma 2.11 Let $X$ be a smooth projective rational surface such that its anti-canonical class is nef.

The self-intersection of a reduced irreducible curve $C$ on $X$ of self-intersection non-positive is either -2 or -1 ; and the following hold:

- $C^{2}=-2$ if and only if $C$ is a smooth rational curve of self-intersection -2 .

- $C^{2}=-1$ if and only if $C$ is a smooth rational curve of self-intersection -1 .

If $T$ is a reduced irreducible curve on the smooth rational surface $X$ such that $T$ is not orthogonal to canonical divisor on $X$, then the following proporties are valid:

Lemma 2.12 With the same notations as above. 
$i-N S_{\mathbb{Q}}(X)=\mathbb{Q} \bar{T} \oplus K_{X}^{\perp}$, in particular if $T$ is a smooth rational curve of self-intersection -1 on $X$, then we have: $N S(X)=\mathbb{Z}[T] \oplus K_{X}^{\perp}$;

$i i$ - the dimension of the $\mathbb{Q}$-vector space $K_{X}^{\perp}$ is $9-K_{X}^{2}$.

Proof. $[i-]$ Let $x \in N S_{\mathbb{Q}}(X)$ :

- $\quad$ if $x \in \mathbb{Q} \bar{T} \cap K_{X}^{\perp}$, we have:

$$
\left\{\begin{array}{l}
\mathrm{x} \cdot \overline{K_{X}}=0 ; \\
x=\alpha \bar{T}, \quad \text { for some } \alpha \in \mathbb{Q} .
\end{array}\right.
$$

Hence $0=\alpha \bar{T} \cdot \overline{K_{X}}$ and consequently $\alpha=0$.

It follows that $\mathbb{Q} \bar{T} \cap K_{X}^{\perp}=\{0\}$;

- on the other hand, we have:

$$
x-\frac{\overline{K_{X}} \cdot x}{K_{X} \cdot T} \bar{T} \in K_{X}^{\perp}
$$

since

$\overline{K_{X}} \cdot\left(x-\frac{\overline{K_{X}} \cdot x}{K_{X} \cdot T} \bar{T}\right)=\overline{K_{X}} \cdot x-\frac{\overline{K_{X}} \cdot x}{K_{X} \cdot T} K_{X} \cdot T=\overline{K_{X}} \cdot x-\overline{K_{X}} \cdot x=0$.

In conclusion, $N S_{\mathbb{Q}}(X)=\mathbb{Q}[T] \oplus K_{X}^{\perp}$.

$[i i-]$ is a consequence of $[i-]$ and the fact that $\rho(X)=10-K_{X}^{2}$.

\subsection{Some linear Algebra Results}

We give here some linear algebra results that we will use in section.

Definition 2.13 Let $n$ be a non-negative integer. Consider two $n$-tuple $Z(t)=\left(z_{1}(t), \ldots, z_{n}(t)\right)$ and $Z^{\prime}(t)=\left(z_{1}^{\prime}(t), \ldots, z_{n}^{\prime}(t)\right)$ of real numbers (resp., $Z(t)=\left(\begin{array}{c}z_{1}(t) \\ \vdots \\ z_{n}(t)\end{array}\right)$ and $Z^{\prime}(t)=\left(\begin{array}{c}z_{1}^{\prime}(t) \\ \vdots \\ z_{n}^{\prime}(t)\end{array}\right)$ of real numbers.

We write $Z(t) \leq Z^{\prime}(t)$ if and only if the inequality $z_{i}(t) \leq z_{i}^{\prime}(t)$ hold for all $i=1, \ldots n$.

Definition 2.14 Let $n$ be a non-negative integer, A family $(Z(t))_{t \in I}$ of $n$-tuple $Z(t)=\left(z_{1}(t), \ldots, z_{n}(t)\right)$ (where $I$ is non empty set) with coefficients in $\mathbb{Q}$, or in $\mathbb{R}$, is bounded if there exists some scalar $b$ such that for every $i$ in $\{1, \ldots, n\}$ and for every $t$ in $I$, the inequality hold: $\left|z_{i}(t)\right| \leq b$, where $|x|$ denotes the absolute value of the scalar $x$.

The next lemma is elementary and the proof is omitted.

Lemma 2.15 Let $A=\left(a_{i, j}\right)$ be an invertible matrix of order $n$ with coefficients in $\mathbb{Q}$, or in $\mathbb{R}$. For every family $(Z(t))_{t \in I}$ (where $I$ is a non empty set), the following assertions are equivalents:

1. the family $(Z(t))_{t \in I}$ is bounded. 
2. the family $(A Z(t))_{t \in I}$ is bounded.

Corollary 2.16 Let $Z(t)=\left(\begin{array}{c}z_{1}(t) \\ \vdots \\ z_{n}(t)\end{array}\right)$ be a family of real numbers. and let $A=\left(a_{i, j}\right)$ be a square matrix of order $n$ with coefficients in $\mathbb{Q}$, or in $\mathbb{R}$, having the following shape:

$$
A=\left(\begin{array}{l|l}
D & 0 \\
\hline 0 & 0
\end{array}\right) \quad \text { (where } D \text { is an invertible matrix of order } n-1 \text { ). }
$$

1. If the family $(Z(t))_{t \in I}$ is bounded, then $(A Z(t))_{t \in I}$ is bounded too.

2. The converse is true if there exist some scalares $d_{1}, \ldots, d_{n}$ such that:

a. $d_{n} \neq 0$

b. for every $t$ in I, the following equality hold:

$$
z_{1}(t)^{2}+\cdots+z_{n-1}(t)^{2}-\sum_{1 \leq i<j \leq n-1} a_{i, j} z_{i}(t) z_{j}(t)=d_{1} z_{1}(t)+\cdots+d_{n-1} z_{n-1}(t)+d_{n} z_{n}(t) .
$$

Proof. Let $Y(t)=A Z(t)$. Then

$$
Y(t)=\left(\frac{D Z^{\prime}(t)}{0}\right)
$$

If we write for every $t \in I, Y(t)$ as $Y(t)=\left(\begin{array}{c}Y^{\prime}(t) \\ y_{n}(t)\end{array}\right)$ and $Z(t)$ as $Z(t)=\left(\begin{array}{c}Z^{\prime}(t) \\ z_{n}(t)\end{array}\right)$, we have for every $t \in I, Y^{\prime}(t)=D Z^{\prime}(t)$ and $y_{n}(t)=0$.

The fact that $(Z(t))_{t \in I}$ is bounded shows that $\left(Z^{\prime}(t)\right)_{t \in I}$ is bounded too; Since $D$ is invertible, the lemma 2.15 shows that $\left(D Z^{\prime}(t)\right)_{t \in I}$ is bounded, that is $\left(Y^{\prime}(t)\right)_{t \in I}$ is bounded, it follows then $(Y(t))_{t \in I}$ is bounded (since $y_{n}(t)=0$ for all $t \in I$.

Conversely, if $(Y(t))_{t \in I}$ is bounded, then $\left(D Z^{\prime}(t)\right)_{t \in I}$ is bounded too, and by using the lemma $2.15,\left(Z^{\prime}(t)\right)_{t \in I}$ is bounded.

The hypothesis $a$. and $b$. confirm that the number $z_{n}(t)$ is a function of $z_{1}(t), \ldots, z_{n-1}(t)$. Hence the family $\left(z_{n}(t)\right)_{t \in I}$ is bounded. In conclusion, $(Z(t))_{t \in I}$ is bounded.

\section{Criterion for a Rational Surface to Be Elliptic}

Let $X$ be a smooth projective rational surface such that the self-intersection of its canonical divisor is zero. Recall that a $(-2)$-curve on $X$ is a smooth rational curve of self-intersection -2 . In this section we prove that if $\left\{C_{1}, \ldots, C_{k}\right\}$ is a set of $(-2)$-curves on $X$ such that the vectors $\left\{\overline{C_{1}}, \ldots, \overline{C_{k}}\right\}$ are linearly dependents in $N S_{\mathbb{Q}}(X)$ (where $k$ is a non-negative integer), then the surface $X$ is elliptic. As a corollary, we prove that the number of $(-2)$-curves on $X$ is bounded 
by 12 ; and this bound is optimal.

Recall some definitions:

let $f: X \longrightarrow B$ be a relatively minimal elliptic surface $X$ over a smooth curve $B$ of genus $g$. By this we always mean the following:

$X$ is a smooth projective surface defined over $\mathbb{C}$ equipped with an elliptic relatively minimal fibration $f: X \longrightarrow B$. That is, $f$ is a surjective morphism satisfying the following conditions:

1- Almost all the fibres are elliptic curves;

2- Each fibre does not contain a (-1)-curve as a component.

If $D=n_{1} C_{1}+\cdots+n_{p} C_{p}$ is the decomposition of the divisor $D$ in reduced irreducible components $C_{1}, \ldots, C_{p}$, then it will be called multiple of multiplicity $m$ if and only if the greatest commun divisor of the integers $n_{1}, \ldots, n_{p}$ is equal to $m$ and $m \geq 2$.

It follows that if $D$ is multiple of multiplicity $m$, then $D=m D^{\prime}$ where $m$ is the multiplicity of $D$ and $D^{\prime}$ is a divisor which is not multiple.

We denote by:

- $\chi$ the Euler characteristic of the surface $X \quad\left(\chi=\chi\left(O_{X}\right)\right)$;

- F a general fiber of $f$;

- $F_{b}$ the fiber of $f$ over the point $b \in B$;

- $F_{b_{1}}=m_{1} F_{1}, \ldots, F_{b_{r}}=m_{r} F_{r}$ the multiple fibers of $f$ of multiplicity $m_{1}, \ldots, m_{r}$ respectively.

Recall that the Néron-Severi group $N S(X)$ of $X$ is the group of divisor classes on $X$ modulo algebraic equivalence. If $C$ is a divisor on $X$, we denote by $[C]$ its class in $N S(X)$ and by $\bar{C}$ the element associated to $[C]$ in $N S_{\mathbb{Q}}(X)$, where $N S_{\mathbb{Q}}(X)=N S(X) \otimes_{\mathbb{Z}} \mathbb{Q}$.

We have ([1, corollaire 12.3 , p. 162]):

$$
\overline{K_{X}}=\left(2 g-2+\chi+r-\Sigma_{i=1}^{i=r} \frac{1}{m_{i}}\right) \bar{F} .
$$

We need the following lemma:

Lemma 3.1 Let $f: X \rightarrow B$ be an elliptic fibration of a smooth projective surface $X$ over a smooth curve B. Then the topological character of a reducible fibre is greater than or equal to the number of irreducible components of this fiber. With equality if and only if this fiber is of type $I_{n}$ (where $n$ is greater than or equal to 2 ).

Arnaud Beauville ([3, the lemma of the page 345]), showed that there exist some inequalities between the genus $g$ of the curve $B$ and the irregularity $q$ of the surface $X\left(q=h^{1}\left(X, O_{X}\right), O_{X}\right.$ 
is the structure sheaf of $X$ ) mainely:

$$
g \leq q \leq g+1
$$

In particular if $X$ is rational, then $q=0$ and the genus $g$ of the curve $B$ is necessarily zero. Thus $B$ is isomorphic to $\mathbb{P}^{1}$. This fact motivates the following definition:

Definition 3.2 An elliptic rational surface is a relatively minimal elliptic rational surface over $\mathbb{P}^{1}$.

The following result is straightforward:

Lemma 3.3 Let $X$ be an elliptic rational surface.

The anti-canonical class of $X$ is nef.

The following result is straightforward:

Lemma 3.4 Let $X$ be an elliptic rational surface.

The anti-canonical class of $X$ is nef.

This is our main result in this section:

Proposition 3.5 Let $X$ be a smooth projective rational surface such that the self-intersection of its canonical divisor is zero.

If there exists a family of (-2)-curves on $X$ such that its elements are linearly dependents in $N S_{\mathbb{Q}}(X)$, then the surface $X$ is elliptic.

Proof. Let $\left\{C_{1}, \ldots, C_{k}\right\}$ be a family of $(-2)$-curves on $X$ such that its elements are linearly dependents in $N S_{\mathbb{Q}}(X)$ ( $k$ is a non-negative integer). We can suppose that there exist some non-negative integers $a_{1}, a_{2}, \ldots, a_{k}$ such that:

$$
\sum_{i=1}^{i=r} a_{i}\left[C_{i}\right]=\Sigma_{j=r+1}^{j=k} a_{j}\left[C_{j}\right]
$$

where $r$ is an integer satisfying $1 \leq r \leq k-1$.

Define $C$ and $C^{\prime}$ as :

$C=\Sigma_{i=1}^{i=r} a_{i} C_{i}$ and $C^{\prime}=\Sigma_{j=r+1}^{j=k} a_{j} C_{j}$.

We have: $0 \geq C^{2}=C . C^{\prime} \geq 0$, then $C^{2}=0=C^{\prime 2}$ and $C$ is linearly equivalent to $C^{\prime}$.

The complete linear system $|C|$ is without fixed components, without base points and by using Stein factorisation gives an elliptic fibration $f: X \longrightarrow \mathbb{P}^{1}$ such that $f$ is relatively minimal.

An immediat consequence is:

Corollary 3.6 Let $X$ be a smooth projective rational surface such that the self-intersection of its canonical divisor is zero.

If the number of (-2)-curves on $X$ is greater than or equal to ten, then $X$ is elliptic. 
Nagata [10, lemme 6.1, p. 292] proved that if $X$ is the blowing-up nine points of the projective plane, then the set of $(-2)$-curves is finite. Here we give another proof of his result in the more general context of smooth projective rational surfaces $X$ such $K_{X}^{2}=0$, where $K_{X}$ is a canonical divisor of the surface. This result is a consequence of the proposition above.

Corollary 3.7 Let $X$ be a smooth projective rational surface such that the self-intersection of its canonical divisor is zero. Then the number of (-2)-curves is less than or equal to 12 , and this bound is optimal.

Proof. Consider a family $\left\{C_{1}, C_{2}, \ldots, C_{k}\right\}$ of $(-2)$-curves on $X$, where $k$ is an integer, Two disjoint possibilities may occur:

If the vectors $\overline{C_{1}}, \overline{C_{2}}, \ldots, \overline{C_{k}}$ are linearly independants in $N S_{\mathbb{Q}}(X)$ which is of dimension 10, then $k \leq 9$.

If not, $X$ admits a relatively minimal elliptic fibration $f: X \longrightarrow \mathbb{P}^{1}$. Recall the equality (see [2] lemme VI.4 page 95 ):

$$
\chi_{t o p}(X)=\chi_{t o p}(B) \chi_{t o p}(F)+\Sigma_{b \in B}\left(\chi_{t o p}(F)\right.
$$

Here, we have:

- $B=\mathbb{P}^{1}$

- $\chi_{\text {top }}(X)$ is the topological character of $X$;

- $\chi_{\text {top }}(F)$ is the topological character of $F$;

- $F_{b}$ is the fiber of $f$ over the point $b \in B$;

- $\chi_{\text {top }}\left(F_{b}\right)$ is the topological character of $F_{b}$ pour $b \in B$.

If $\chi$ is the Euler characteristic of the surface $X$, the Noether formula is:

$$
12 \chi=K_{X}^{2}+\chi_{\text {top }}(X) \quad\left(\chi=\chi\left(O_{X}\right)\right) .
$$

Since $K_{X}^{2}=0$ and $\chi_{t o p}(F)=0$ (since $F$ is an elliptic curve) we have:

$$
12 \chi=\Sigma_{b \in B} \chi_{t o p}\left(F_{b}\right)
$$

We denote by $F_{1}, F_{2}, \ldots, F_{t}$ the reducible fibers of $f$. We have for every $i=1,2, \ldots, t$ :

$$
l_{i} \leq \chi_{\text {top }}\left(F_{i}\right) \text {, where } l_{i} \text { is the number of irreducible components of } F_{i} .
$$


Which gives

$$
\sum_{i=1}^{i=t} l_{i} \leq 12 \chi
$$

Hence the number of $(-2)$-curves is less or equal than $12 \chi=12$ (since $X$ is rational).

The number of $(-2)$-curves of the extremal jacobian elliptic rational surface $X_{3333}$ ([9, Tableau 5.3, page 549]) is 12, which ends the proof.

\section{Some Rational Surfaces Having an Infinite Number of (-1)- Curves}

In what follows a $(-1)$-divisor $D$ on a smmoth projective surface $Z$ is a divisor on $Z$ such that $D^{2}=-1=D \cdot K_{Z}$, where $K_{Z}$ is a canonical divisor on $Z$. We need the following result:

Proposition 4.1 Let $X$ be a smooth projective rational surface such that $K_{X}^{2}=0$. Suppose that the intersection form is negative definite on the space spanned over the field of rational numbers by the $\overline{C_{i}}, i=1 \ldots, l$, where the $C_{i}$ are $(-2)$-curves on $X$.

Then there exists an infinite number of $(-1)$-divisors such that each $(-1)$-divisor intersect positively each $C_{i}, i=1, \ldots, l$.

Proof. The hypothesis "the intersection form is negative definite on the space spanned by the $\overline{C_{i}}, i=1, \ldots, l$ " shows that the vectors $\left\{\overline{C_{i}}, i=1, \ldots, l\right\}$ are linearly independents in the orthogonal of the canonical divisor of $X$. In particular, $l$ is less than or equal to 8 . For a large integer $n$ (e.g., $n \geq E_{0} . C_{j}$, for all $j=1, \ldots, l$, where $E_{0}$ is a fixed (-1)-curve), we consider the unique divisor $\Delta_{n}$ whose support is in the set of $(-2)$-curves $C_{i}, i=1, \ldots, l$ and defined by:

$$
\Delta_{n} \cdot C_{j}=|d|\left(n-E_{0} \cdot C_{j}\right)
$$

for each $j=1, \ldots, l$, where $d$ is the determinant of the matrix $\left(C_{i} . C_{j}\right)_{1 \leq i, j \leq l}(|d|$ is the absolute value of $d$ ).

The divisor $E_{n}$ defined for large $n$ by:

$$
E_{n}=E_{0}+\Delta_{n}-\left(2 E_{0} \cdot \Delta_{n}+\Delta_{n}^{2}\right)\left(-K_{X}\right)
$$

is a (-1)-divisor intersecting positively each $C_{i}, i=1, \ldots, l$. And for $E_{n} \neq E_{m}$ for $n \neq m$.

Proposition 4.2 Let $X$ be a smooth projective rational surface such that the anti-canonical class is nef and of self-intersection zero.

If the intersection form is negative definite on the space spanned by $\bar{C}_{i}$, where the $C_{i}$ constitute a connected component of the set of all $(-2)$-curves on $X$, then the number of $(-1)$-curves on $X$ is infinite. 
Proof. By hypothesis we can find a connected component $\left\{C_{1}, \ldots, C_{l}\right\}$ of the family of all $(-2)$-curves on $X$ such that the intersection form is negative definite on the space spanned by the $\overline{C_{i}}, i=1, \ldots, l$. An application of the proposition above yields to the existence of an infinite family $E_{n}$ of $(-1)$-divisors such that $E_{n} . C_{i} \geq 0$. By construction the element $E_{n}$ is equal to $E_{n}=E_{0}+\Delta_{n}-\left(2 E_{0} \cdot \Delta_{n}+{\Delta_{n}}^{2}\right)\left(-K_{X}\right)$ where the support of $\Delta_{n}$ is in the set $C_{i}, i=1, \ldots, l$ and $E_{0}$ is a fixed (-1)-curve on $X$.

For each (-2)-curve $C$ different from $C_{i}, i=1, \ldots, l$, we have $C . C_{i}=0$ for all $i=1, \ldots, l$; then $C . E_{n}=C . E_{0} \geq 0$. Consequently $E_{n}$ intersect every (-2)-curve on $X$ positively. Hence by [7, Theorem, p.3], $E_{n}$ is irreductible.

\section{$5 \quad$ Proof of Theorem 1.1}

Let $s$ be the rank of the matrix $\left(C_{i} . C_{j}\right)_{i, j=1, \ldots, r}$, where $\left\{C_{i} ; i=1, \ldots, r\right\}$ is the finite set of $(-2)$-curves on $X$.

Suppose that $X$ is extremal, we claim that $s=8$.

Proof of the claim:

If $s=0$, i.e., $X$ has no $(-2)$-curves, then the nine points are in general position and therfore $X$ has an infinite number of $(-1)$-curves.

If $s \in\{1, \ldots, 7\}$, then we can find a divisor $D$ such that the following conditions hold:

1. $D^{2}<0$,

2. D. $K_{X}=0$,

3. D. $C_{i}=0$, for each $i=1, \ldots, r$.

Then fix a (-1)-curve $E_{0}$, and for each integer n, consider the (-1)-divisor $E_{n}$ defined as follows:

$$
E_{n}=E_{0}+n D-\left(n E_{0} \cdot D+\frac{n^{2}}{2} D^{2}\right)\left(-K_{X}\right)
$$

By [7, Theoerem, p.3] $E_{n}$ is a (-1)-curve. Two distinct integers $\mathrm{n}$ and $\mathrm{m}$ give two distinct $(-1)$-curves $E_{n}$ and $E_{m}$; so $X$ has an infinite number of (-1)-curves.

Hence $s=8$ is a necessarily condition in order that $X$ to be extremal.

In what follows $s$ is equal to 8 . We will distinguish between two cases:

1. First Case: If the vector space over the field of rational numbers spanned by the set of $(-2)$-curves is not equal to the orthogonal of the canonical divisor, then according to the proposition 4.2 ( page 12), $\mathrm{X}$ will have an infinite number of $(-1)$-curves.

2. Second Case: If the vector space over the field of rational numbers spanned by the set of $(-2)$-curves is equal to the orthogonal of the canonical divisor, then we will distinguish between the case where $X$ is elliptic or not: 
First subcase: If $X$ is not elliptic, then according to proposition $3.5(10)$, the $(-2)$ curves are linearly independents over the field of rational numbers; so the number of $(-2)$-curves on $X$ is nine. Two cases comes:

- if the set of $(-2)$-curves is connected, then $-K_{X}$ is a linear combination of strictly positive integers of all $(-2)$-curves.

- If the set of $(-2)$-curves is not connected, then $-\bar{K}_{X}$ is not a linear combination of strictly positive rational numbers of all $(-2)$-curves. Applying proposition 4.2 (p. 12) to any connected component of the set of (-2)-curves for which the intersection form is negative definite gives the fact that $X$ contains an infinite number of $(-1)$-curves.

Second subcase: If $X$ is elliptic, then $-\bar{K}_{X}$ is a linear combination of strictly positive rational numbers of all $(-2)$-curves.

In conclusion if $X$ is extremal, then it satisfies the two conditions below:

a. the rank of the matrix $\left(C_{i} . C_{j}\right)_{i, j=1, \ldots, r}$ is equal to 8 , where $\left\{C_{i} ; i=1, \ldots, r\right\}$ is the finite set of $(-2)$-curves on $X$;

b. There exist $r$ strictly positive rational numbers $a_{i}, i=1, \ldots, r$ such that $-\bar{K}_{X}=$ $\sum_{i=1}^{i=r} a_{i} \bar{C}_{i}$.

Conversely, the fact that the rank of the matrix $\left(C_{i} \cdot C_{j}\right)_{i, j=1, \ldots, r}$ is equal to eight enables us to suppose for example that the vectors $\overline{C_{1}}, \ldots, \overline{C_{8}}$ are linearly independents in $N S_{\mathbb{Q}}(X)$ and the intersection form (induced by the intersection form on $K_{X}^{\frac{1}{X}}$ ) is negative definite on the vector space over the field of rational numbers spanned by the vectors $\overline{C_{1}}, \ldots, \overline{C_{7}}$ and $\overline{C_{8}}$.

Since $-K_{X}$ is not trivial and of self-intersection zero, the vectors $\overline{C_{1}}, \ldots, \overline{C_{8}}$ and $\overline{-K_{X}}$ are linearly independents in $N S_{\mathbb{Q}}(X)$, in particular

- the elements $\left[C_{1}\right], \ldots,\left[C_{8}\right]$ and $\left[-K_{X}\right]$ are linearly independents in $N S(X)$;

- $\quad$ the vecor space $<\overline{C_{1}}, \ldots, \overline{C_{8}}, \overline{-K_{X}}>$ is equal to $K_{X}^{\perp}$.

It is easy to prove the following lemma:

Lemma 5.1 Let $E$ be a (-1)-curve on $X$. Then the equality

$-\bar{K}_{X}=\sum_{i=1}^{i=r} a_{i} \bar{C}_{i}$ gives rise the inequalities:

$$
0 \leq E . C_{i} \leq \frac{1}{a_{i}}
$$

for every $i=1, \ldots, r$.

Let $E_{0}$ be a fixed (-1)-curve on $X$ and consider a (-1)-curve $E$ on $X$. There exist some rational numbers $\alpha_{1}(E), \ldots, \alpha_{9}(E)$ such that:

$$
\bar{E}=\overline{E_{0}}+\alpha_{1}(E) \overline{C_{1}}+\cdots+\alpha_{8}(E) \overline{C_{8}}+\alpha_{9}(E)\left(\overline{-K_{X}}\right) .
$$


We claim that:

the numbers $\alpha_{1}(E), \ldots, \alpha_{9}(E)$ may be taken such that their denominators are bounded. In fact, let $M$ be the lattice of $N S(X)$ defined by the free family $\left\{\left[C_{1}\right], \ldots,\left[C_{8}\right],\left[-K_{X}\right]\right\}$, i.e., $\left.M=\mathbb{Z} \bar{C}_{1} \oplus \ldots \oplus \mathbb{Z} \bar{C}_{8} \oplus \mathbb{Z} \overline{\left(-K_{X}\right)}\right)$. The set $\left\{\bar{E}-\bar{E}_{0} / E\right.$ est une courbe $(-1) \operatorname{sur} X\}$ is a sbset of $N S(X)$ and of $K_{X}^{\perp}$; if $M^{+}$denotes $\{x \in N S(X) /$ pour un certain entier naturel non nul $n, n x \in M\}$, then $M^{+}=N S(X) \cap K_{X}^{\perp}$. Since $\frac{N S(X)}{M}$ is a finitely generated group, the group $\left(\frac{N S(X)}{M}\right)_{t}$ whose elements are the torsion elements of $\frac{N S(X)}{M}$ is finite ([8, Theorem 8.5., p.46]). Since $\left(\frac{N S(X)}{M}\right)_{t}=\frac{M^{+}}{M}$, there exists $N \in \mathbb{N}, N \neq 0, \forall x \in M^{+}$ $N x \in M$; in particular there exists some non-negative integer $N$ such that for every $(-1)$-curve $E N\left(\bar{E}-\bar{E}_{0}\right) \in \mathbb{Z} \bar{C}_{1} \oplus \ldots \oplus \mathbb{Z} \bar{C}_{8} \oplus \mathbb{Z} \overline{\left(-K_{X}\right)}$.

According to the lemma 5.1 (p. 14) we have for all $i=1,2, \ldots, 9$ :

$$
-E_{0} \cdot C_{i} \leq \sum_{j=1}^{j=8} C_{i} \cdot C_{j} \alpha_{j}(E) \leq \frac{1}{a_{i}}-E_{0} \cdot C_{i} .
$$

In matrix form:

$$
\left(\begin{array}{c}
-E_{0} \cdot C_{1} \\
\vdots \\
-E_{0} \cdot C_{8} \\
0
\end{array}\right) \leq\left(\begin{array}{c|c}
D & 0 \\
\hline 0 & 0
\end{array}\right)\left(\begin{array}{c}
\alpha_{1} \\
\vdots \\
\vdots \\
\alpha_{8} \\
\alpha_{9}
\end{array}\right) \leq\left(\begin{array}{c}
\frac{1}{a_{1}}-E_{0} \cdot C_{1} \\
\vdots \\
\frac{1}{a_{8}}-E_{0} \cdot C_{8} \\
0
\end{array}\right)
$$

where $D=\left(C_{i} \cdot C_{j}\right)_{1 \leq i, j \leq 8}$.

The equality $E^{2}=-1$ gives

$\alpha_{1}(E)^{2}+\cdots+\alpha_{8}(E)^{2}-\sum_{1 \leq i<j \leq 8} C_{i} \cdot C_{j} \alpha_{i}(E) \alpha_{j}(E)=E_{0} \cdot C_{1} \alpha_{1}(E)+\cdots+E_{0} \cdot C_{8} \alpha_{8}(E)+\alpha_{9}(E)$.

Since $D$ is a negative definite square matrix of order eight, it is invertible and an application of corollary 2.16 (p. 8) gives the fact that $\left(\left(\alpha_{1}(E), \ldots, \alpha_{8}(E), \alpha_{9}(E)\right)\right)_{E}$ is bounded.

Acknowledgments: This work was done at Laboratoire J. A. Dieudonné, U.M.R. C.N.R.S. N: 6621, Université de Nice Sophia-Antipolis (Nice, France), and was written at the Abdus Salam International Centre for Theoretical Physics. Many thanks to Professors Gang Xiao, André Hirschowitz and Charles Walter for many useful discussions. I would like also to acknowledge the hospitality of the Abdus Salam International Centre for Theoretical Physics, without which this work would not have been possible. 


\section{References}

[1] Barth W. , Peters C. , Van de Ven. A. Compact Complex Surfaces. Berlin, Springer (1984).

[2] Beauville, A. Surfaces algébriques complexes, Astérisque, vol. 54, Société Mathématique de France, Paris, 1978.

[3] Beauville, A. L'inégalité $p_{g} \geq 2 q-4$ pour les surfaces de type général, Appendice à , Debarre O. : “Inégalités numériques pour les surfaces de type général ”, p. 319-346, Bulletin de la SMF, tome 110 , fasc. 3 (1982).

[4] Hartshorne R. Algebraic Geometry, Graduate Texts in Mathematics, Springer Verlag (1977).

[5] Kodaira K., On compact complex analytic surfaces, I, Ann. of Math. 71(1960), pp. 111152.

[6] Kodaira K., On compact analytic surfaces II-III, Ann. of Math. 77 (1963), pp. 563-626; IV, Ann. of Math. 78 (1963), pp. 1-40.

[7] Lahyane M., Irreducibility of the (-1)-Classes on Smooth Rational Surfaces, Preprint of the Abdus Salam International Centre for Theoretical Physics, Trieste, Italy (August 2001).

[8] Lang, S. Algebra, Addison-Wesley Publishing Company, Inc. 3rd. Ed. (1993).

[9] Miranda R.- Persson U. On Extremal Rational Elliptic Surfaces. Math. Z. 193, 537-558 (1986).

[10] Nagata M. On rational surfaces, II, Mem. Coll. Sci. Univ. Kyoto, Ser. A Math. 33 (1960), 271-293.

[11] Rosoff, J.A. Effective divisor classes and blowings-up of $P^{2}$. Pacific Journal of Mathematics Vol. 89, No.2,1980.

[12] Siegel, C. L. Meromorphe Funktionen auf kompakten analytishen Mannigfaltigkeiten. Nachr. Akad. Wiss. Göttingen, 1955, pp. 71-77. 